\title{
NOTAS SOBRE LOS NIVELES PROVINCIALES DE BIENESTAR SOCIAL
}

\author{
$311: 339.11(46)$
}

por

Ignacio Ballester Ros

SUMARIO: I. INTRODUCCION.-II. LOS NIVELES DE: 1. POBLACIÓN. 2. Atenciones sanitarias. 3. Actividad laboral. 4. Capacidad de conSumo. 5. Prom oción cultural. 6. Convivencia social. 7. EsparcimienTO. 8. EOUIPAMIENTO DE LOS H OGARES.-III. NIVEL CONJUNTO DE BIENESTAR SOCIAL. - IV. ANEXO: INDICADORES DE CONJUNTO Y DE GRUPO.

\section{INTRODUCCION}

El Servicio Sindical de Estadística ha elaborado en el pasado mes de septiembre un estudio sobre "Niveles de bienestar social. Ensayo sobre indicadores provinciales", que se ha publicado recientemente.

Como base de dicho estudio se ha tomado el programa de la O. C. D. E. para el establecimiento de un plan de indicadores sociales, teniendo en cuenta «los criterios a partir de los cuales se pueden evaluar las necesidades, definir los objetivos y medir los resultados de las acciones emprendidas".

El bienestar social se concreta, según este programa, en « 24 preocupaciones especiales» que dependen de ocho «dominios objeti- 
vos»: salud, expansión de la personalidad por adquisición de conocimientos, empleo y calidad de la vida de trabajo, tiempo y esparcimiento, bienes y servicios disponibles, contorno físico, seguridad de las personas y administración de justicia y participación en la vida colectiva.

Consciente de las limitaciones de los datos disponibles, a nivel provincial, y de las dificultades inherentes al propósito de medir cuantitativamente el bienestar social, que inicialmente requiere «la igualdad de oportunidades, extendida a todos lós bienes, materiales e inmateriales, que se consideren socialmente deseables", el Servicio Sindical de Estadística ha calificado modestamente este trabajo como un ensayo, punto de arranque que ha de permitir perfeccionamientos posteriores, así como la oportunidad de profundizar en estudios monográficos provinciales, el primero de los cuales, dedicado a la Provincia de Madrid, acaba también de aparecer.

Se ha partido en el trabajo que contemplamos de la "correspondencia entre el bienestar y sus manifestaciones externas, tanto de causa como de efecto".

El «conjunto de manifestaciones externas» del bienestar se ha concretado en 94 variables, sistematizadas en los ocho grupos siguientes:

- Población.

- Atenciones sanitarias.

- Actividad laboral.

- Capacidad de consumo.

- Promoción cultural.

- Convivencia social.

- Esparcimiento.

- Equipamiento de los hogares.

Con referencia a cada una de las variables, se presentan en la monografía:

- Las cifras absolutas correspondientes al conjunto nacional y a cada Provincia.

- Los valores relativos igualmente correspondientes al conjunto nacional y a cada Provincia. 
- Los indicadores provinciales de cada variable en relación con el conjunto nacional, al que se asigna, en todo caso, el valor de 100 .

- Los indicadores provinciales de cada uno de los ocho grupos enumerados, resumen y concreción de los valores provinciales de las variables contempladas en cada grupo, presentados en forma análoga a los indicadores provinciales de cada variable.

- El indicador conjunto provincial de bienestar social, que expresa "una valoración comparativa entre los niveles de grupo, alcanzados en las distintas Provincias, y el conjunto nacional».

Se dan a conocer las fuentes utilizadas en la elaboración del estudio, constituidas, en su mayor parte, por las estadísticas elaboradas por el Instituto Nacional de Estadística, y se insertan unas amplias observaciones a los resultados obtenidos, que permiten subrayar las diferencias interprovinciales.

Dada la naturaleza del tema y la riqueza informativa - de la que, por falta de espacio, se transcriben solamente los indicadores provinciales de niveles de grupos y del conjunto- que contiene este estudio en orden al análisis del distinto grado relativo de bienestar social de las Provincias españolas, resulta de notable interés la redacción de un comentario en el que resalten las características de mayor significación.

\section{LOS NIVELES DE:}

\section{Población}

Las variables que se tienen en cuenta en este grupo y los valores correspondientes al conjunto nacional, que sirven de base para la elaboración de los indicadores provinciales, son:

Población de hecho que vive en zona urbana

Por 1.000 habitantes ...

Idem 343,1 


\begin{abstract}
Movimiento natural de la población en el año
\end{abstract}

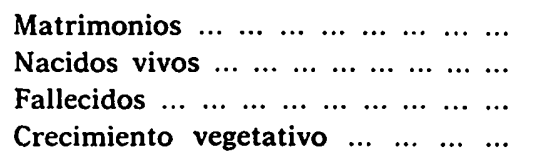

267.171

Por 1.000 habitantes ......

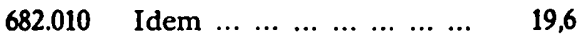

$\begin{array}{lllllllllr}294.567 & \text { Idem } & \ldots & \ldots & \ldots & \ldots & \ldots & \ldots & \ldots & 8,5\end{array}$

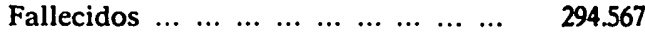

Idem $\begin{array}{llllllll} & \ldots & \ldots & \ldots & \ldots & \ldots & \ldots & \ldots\end{array} \quad 11,2$

\title{
Movimientos migratorios
}

Emigrantes al extranjero $\ldots \ldots \ldots \ldots$

Emigrantes a otras Provincias ... ...

\section{Habitantes de hecho de diez y más} años
Residentes en la Provincia y que en 1960 residian fuera de ella ...
No residentes en la Provincia y que sí residian en ella en 1960 ...

Idem

La densidad de población española se caracteriza por su desigual distribución geográfica. De las 20 Provincias con densidad superior al promedio nacional, 18 -Pontevedra y La Coruña; Oviedo y Santander; Vizcaya y Guipúzcoa; Gerona, Barcelona, Tarragona y Baleares; Valencia, Alicante y Murcia; Málaga, Sevilla y Cádiz, y las Canarias - son marítimas, y tan sólo Madrid y Alava son interiores. Son de señalar los altos niveles de saturación alcanzados por Barcelona (539 habitantes $/ \mathrm{km}^{2}$ ), Madrid (504), Vizcaya (498) y Guipúzcoa (331). Salvo cinco costeras, las 30 Provincias restantes, con densidades menores, se hallan en el interior del país, y entre ellas figuran 11, que pueden calificarse de desérticas -Cáceres, Avila, Zamora, Palencia, Segovia, Soria, Guadalajara, Teruel, Cuenca y Albacete- por registrar densidades inferiores a 25 habitantes/kilómetro cuadrado. Ello es expresión de la concentración de la población en las zonas litorales y marítimas, de su disminución en las zonas deprimidas y del desequilibrio entre ambias zonas.

El proceso de urbanización se pone de manifiesto por el hecho de que más de la mitad de la población vive en zona urbana, fenómeno que se acentúa en 13 Provincias: las tres Vascongadas; Barcelona, Valencia y Alicante; Zaragoza; Madrid y Valladolid, y Córdoba, Sevilla, Málaga y Cádiz. Las tasas más elevadas corresponden a Madrid, Barcelona y Cádiz, y las más bajas, a algu- 
nas Provincias deprimidas. En el período 1960-70, en que el éxodo rural fue muy intenso, las dos terceras partes de los Municipios españoles disminuyeron de población, fenómeno más acusado con respecto a las entidades singulares de población que, en ciertas áreas, quedaron, en muchos casos, despobladas. La urbanización, por sí sola, no produce el bienestar; pero el ruralismo dificulta su adquisición:

La población comprendida entre quince y treinta y nueve años, fuerza potencial de trabajo que no ha alcanzado aún la edad madura, influida por los movimientos natural y migratorio de la población, resulta un fenómeno atípico que afecta positivamente tanto a Provincias de alto grado de concentración urbana como a otras deprimidas, marítimas o interiores.

Con referencia al movimiento natural de la población, señalamos, en primer término, que las tasas más altas de nupcialidad corresponden a las Provincias de la zona marítima, enumeradas en relación con la densidad de población, con las excepciones de Oviedo, Vizcaya y Guipúzcoa y la inclusión de Almería; y a Madrid, Logroño y Cáceres en el interior. El valor mínimo es el de Guadalajara.

Las tasas más elevadas de natalidad se distribuyen en forma similar a las de densidad y nupcialidad, si bien destacan solamente Pontevedra y Vizcaya al Norte; las del litoral mediterráneo, a las que hay que añadir Almería y Granada, con las excepciones de Gerona y Tarragona; y en el interior, Madrid y Valladolid.

Las altas tasas de mortalidad, superiores al promedio nacional, más generalizadas, se extienden a 35 Provincias, que cubren Galicia, Asturias, León y Castilla la Vieja, Extremadura y Castilla la Nueva, Navarra, Aragón, Cataluña y Baleares y las Provincias de Málaga, Córdoba y Huelva. Se exceptúan Valladolid, Santander, Madrid, Zaragoza y Barcelona, que junto con las Vascongadas, Cádiz y las Canarias presentan, por el contrario, las tasas más bajas.

El crecimiento vegetativo se manifiesta en forma parecida a la densidad, natalidad y nupcialidad. Las tasas máximas corresponden a Pontevedra, las Vascongadas, Barcelona, Alicante, Murcia, Almería y Granada, Málaga, Sevilla y Cádiz, y las Canarias en la zona marítima, y a Madrid y Valladolid en el interior. Las Palmas, con la tasa más alta, cercana al 20 por 1.000 , presenta un proceso de superpoblación, sin más solución que la emigración, si no se modifica su actual estructura económica y social. Las tasas mínimas corresponden a las Provincias deprimidas. 
La emigración al extranjero procede especialmente de: a) Galicia y el Valle del Duero - León, Zamora, Salamanca y Avila-. b) Submeseta Sur -Extremadura y Albacete-c) Sur -Almería, Granada, Jaén, Córdoba, Málaga y Santa Cruz de Tenerife-. La emigración interior a otras Provincias, reflejada en las tasas de emigrantes totales, y de diez y más años no residentes en cada Provincia y que sí residían en 1960 , por 1.000 habitantes, de más amplia base, procede de: a) Lugo y gran parte del Valle del Duero. b) Submeseta Sur, excepto Madrid. c) Valle del Ebro -Logroño, Huesca, Lérida y Teruel-; y d) Andalucía —Jaén, Granada, Córdoba, Cádiz y Huelva-. Y se dirige principalmente, según se desprende de las tasas por 1.000 habitantes de los inmigrantes totales y de diez y más años residente en cada Provincia que en 1960 residían fuera, a: a) Vascongadas, Navarra y Zaragoza. $b$ ) Cataluña - menos Lériday Baleares. c) Región valenciana. d) Madrid y Valladolid. Las principales Provincias receptoras de inmigrantes en 1960-70 son: Madrịd y Barcelona, con más de 600.000 , y Valencia y Zaragoza, con más de 100.000.

Los movimientos migratorios reflejan los desequilibrios provinciales de bienestar. El «hombre hace su propia evaluación del nivel relativo de bienestar, y con su actitud es el protagonista de las posibilidades de desarrollo de un área geográfica». La inmigración provoca un «empobrécimiento irreversible en los recursos humanos que bloquea las posibilidades de elevar el bienestar social».

El indicador de grupo, transcrito en el Anexo, que resume y concreta las características analizadas, perfila los siguientes focos de bienestar, que se extienden a 14 Provincias: a) Las Vascongadas y Santander. b) Barcelona. c) Alicante, Valencia y Murcia. d) Cádiz y Sevilla. e) Canarias; y f) Madrid y Valladolid.

\section{ATENCIONES SANITARIAS}

Las variables que se tienen en cuenta en este grupo y los valores correspondientes al conjunto nacional, que sirven de base para la elaboración de los indicadores provinciales son: 
Gastos. anuales de los hogares en servicios médicos y conservación de la salud (millones de ptas.) $\ldots \ldots \ldots \ldots$.....

Médicos colegiados:

Residentes en la capital de la Pro-

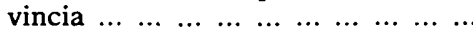

No residentes en la capital de la Provincia

34.951 Por 100.000 habitantes ... 100,6

17.065 Idem

49,1

Odontólogos y estomatólogos colegiados:

Residentes en la capital de la Pro$\begin{array}{lllllllllll} & \text { vincia } & \ldots & \ldots & \ldots & \ldots & \ldots & \ldots & \ldots & \ldots & \ldots\end{array}$

No residentes en la capital de la

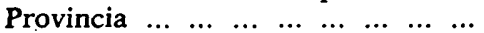

Practicantes colegiados:

Residentes en la capital de la Pro-

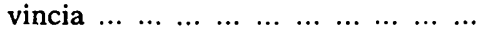

No residentes en la capital de la $\begin{array}{lllllllll}\text { Provincia } & \ldots & \ldots & \ldots & \ldots & \ldots & \ldots & \ldots & \ldots\end{array}$

Camas en centros sanitarios

65.516 Pesetas/persona 1.886

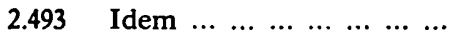

1.171 Idem ..

$\begin{array}{llllllllll}16.437 & \text { Idem } & \ldots & \ldots & \ldots & \ldots & . . & \ldots & \ldots & 47,3\end{array}$

$\begin{array}{llllllllll}11.559 & \text { Idem } & \ldots & \ldots & \ldots & \ldots & \ldots & \ldots & \ldots & 33,6\end{array}$

$\begin{array}{llllllllll}177.385 & \text { Idem } & \ldots & \ldots & \ldots & \ldots & \ldots & \ldots & \ldots & 510,6\end{array}$

\section{Seguridad Social}

Médicos

41.728

Idem

Intervenciones quirúrgicas anuales.

Población protegida

1.272 .855

Por 1.000 habitantes ... ...

Por 100 habitantes ... ...

116.865 Miles de ptas/población $\begin{array}{lllllll}\text { protegida } & \ldots & \ldots & \ldots & \ldots & 4,2\end{array}$

Recaudación cuotas régimen general (millones de ptas.)

El indicador de grupo, que figura en el Anexo, refleja los valores más altos, que corresponden a: a) Oviedo, Santander, Vascongadas, Navarra y Zaragoza. $b$ ) Barcelona y Tarragona. c) Castellón y Valencia. d) Sevilla. e) Madrid y Guadalajara. Una vez más con amplio predominio de las Provincias litorales.

El estado de salud de la población es vital en una sociedad. Carecemos de indicadores directos que midan la salud y la bondad de los servicios sanitarios. Sólo disponemos de indicadores del cos- 
te, del personal profesional, de la capacidad de los establecimientos y del alcance de los servicios.

No es fácil interpretar las diferencias interprovinciales del gasto de los hogares. Las tasas más altas corresponden a: Orense, Santander, Alava y Vizcaya; Navarra, Logroño y Zaragoza, Huesca y Lérida; Gerona, Barcelona y Tarragona; Valencia y Alicante; Madrid, Cuenca, Segovia y Soria, y las islas Canarias, Provincias marítimas o interiores, industrializadas o agrarias, con grandes urbes o rurales.

La distribución provincial de los médicos no es homogénea. Dos de cada tres residen en capitales, y uno en el resto de la Provincia; si bien sólo 12 Provincias - Santander, Alava, Vizcaya y Navarra, Zaragoza, Barcelona, Valencia, Granada y Sevilla, Madrid, Salamanca y Valladolid- superan la media nacional, en el primer caso, y 32 en el segundo. La concentración en las capitales no es paralela al desarrollo económico y social, y parece obedecer a causas muy diversas. Madrid da el máximo nacional en la capital y el mínimo en la Provincia.

Tradicionalmente se considera el número de odontólogos como indicador válido del desarrollo sanitario. La proporción entre capitales y el resto de la Provincia es similar a la de médicos, e igualmente coincide, en líneas generales, la distribución de las tasas más altas, correspondientes a 13 Provincias.

Los practicantes presentan una distribución más equilibrada, y aparecen en número relativamente mayor en las zonas rurales, en las que hay escasez de médicos.

Es escaso el número de camas disponibles en los establecimientos sanitarios, y su distribución es similar, en general, a la de los gastos de los hogares.

Las intervenciones quirúrgicas, la población protegida, el coste de la asistencia y la recaudación de cuotas de la Seguridad Social presentan, en general, sus más altas tasas en las Provincias más industrializadas, aunque no hay una franca correlación entre estas características, el personal sanitario disponible, la calidad de las prestaciones y la amplitud de los servicios de que se dispone en cada Provincia. 


\section{ACTIVIDAD LABORAL}

Las variables que se tienen en cuenta en este grupo y los valores correspondientes al conjunto nacional, que sirven de base para la elaboración de los indicadores provinciales, son:

\section{Cifras absolutas}

Población económicamente activa:

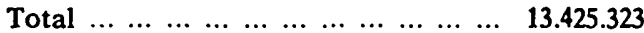

Menor de cuarenta y cinco años $\ldots . \quad 8.484 .125$

Por 100 habitantes ... ...

Por 100

Mujeres trabajadoras:

\section{Valores relativos}

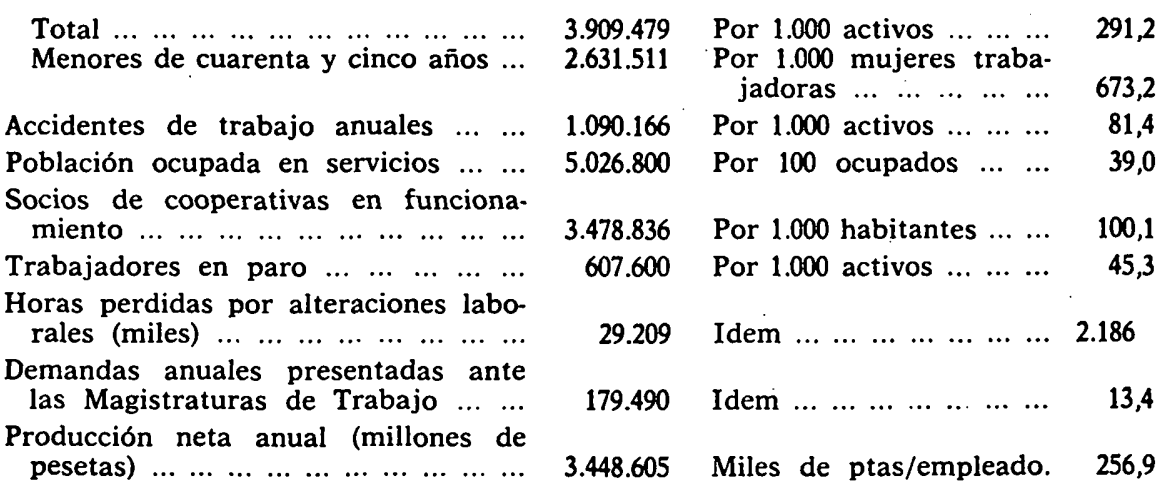

El indicador de grupo, expuesto en el Anexo, refleja los valores más altos, que corresponden a: $a$ ) Galicia. $b$ ) Alava y Vizcaya. c) Cataluña y Baleares. d) Región valenciana y Murcia. e) Santa Cruz de Tenerife; y $f$ ) Madrid y Ciudad Real. Igualmente con un claro predominio de Provincias litorales y más amplio que los anteriores, por cuanto que comprende Provincias de economía básicamente agraria.

La «actividad laboral» es una de las formas básicas de participación del hombre en la sociedad por la que «se integra como miembro pleno de la comunidad". El trabajo se convierte así en uno de los componentes básicos del bienestar social.

La población económicamente activa mide la fuerza de trabajo disponible en el país por sectores económicos - agrario, industrial y de servicios-, y su fuente más fidedigna es la Encuesta de Pobla- 
ción Activa del Instituto Nacional de Estadística. Las tasas de activos por 100 habitantes, superiores al promedio nacional, alcanzan 22 Provincias, que se distribuyen de esta forma: a) Galicia, Oviedo, Santander, Guipúzcoa y Alava. b) Logroño, Huesca, Lérida y Teruel. c) Gerona, Barcelona, Baleares, Tarragona, Castellón y Alicante. d) Burgos, León y Zamora. $e$ ) Toledo. El sector primario está representado por la actividad agraria o extractiva en los Valles del Ebro y Duero y en Galicia.

La distribución de la población activa menor de cuarenta y cinco años difiere parcialmente de la anterior, y las Provincias con tasas superiores a la media nacional son: a) Vascongadas, Navarra y Zaragoza. b) Barcelona. c) Valencia, Alicante y Murcia. d) Almería, Córdoba, Sevilla, Málaga y Cádiz. e) Canarias; y $f$ ) Madrid y Ciudad Real. Las Palmas dispone de población joven en razón de su elevada fecundidad. Las demás Provincias enumeradas deben su situación favorable a su crecimiento vegetativo o a su fuerte inmigración. Por el contrario, Provincias de acusada emigración, como Teruel, Soria, Lugo y Guadalajara, registran un envejecimiento de su población activa.

La población activa femenina alcanza sus valores máximos en 14 Provincias: a) Galicia, Oviedo, Santander, León y Zamora, donde se dedica preferentemente al campo. b) Gerona, Baleares y Alicante, trabajando especialmente en el sector servicios; y $c$ ) Madrid, Ciudad Real y Toledo. Las trabajadoras menores de cuarenta y cinco años sobresalen en un área más amplia, que se extiende a 27 Provincias, y es fruto del proceso de incorporación de la mujer al trabajo fuera del hogar, incluso en el sector industrial.

La población ocupada en el sector servicios se concentra principalmente en las grandes urbes o en los Municipios turísticos de las 13 Provincias siguientes: Vizcaya y Zaragoza; Gerona, Barcelona, Baleares y Valencia; Sevilla, Málaga y Cádiz; Canarias, y Madrid y Valladolid.

La producción neta de los tres sectores de la actividad económica presenta sus valores máximos en 20 Provincias, que pueden agruparse en esta forma: a) Oviedo, Santander, Vascongadas y Navarra, Zaragoza, Huesca y Lérida. b) Gerona, Barcelona, Baleares, Tarragona, Valencia y Alicante. c) Huelva. d) Las Palmas, y e) Madrid y Valladolid.

Los accidentes de trabajo son más numerosos en las Provincias industriales, con abundante mano de obra y mayores riesgos en el 
uso de la maquinaria. Las demandas presentadas ante las Magistraturas de Trabajo destacan igualmente por su número en las zonas industriales. El cooperativismo se ha désarrollado, de manera especial, en las Provincias predominantemente agrarias de los Valles del Duero y Ebro, Cataluña, Región valenciana y Castilla la Nueva, aparte de algunas andaluzas. El paro es un fenómeno predominante en el Sur del país. Extremadura, Andalucía y Canarias son Regiones en que el paro es endémico. Igualmente registra cierta importancia en Madrid, Toledo y Ciudad Real. La pérdida de horas de trabajo, por conflictos laborales, ha afectado especialmente a las Provincias más industrializadas (Vascongadas y Navarra; Oviedo, Barcelona y Valladolid).

\section{CAPACIDAD DE CONSUMO}

Las variables que se tienen en cuenta en este grupo y los valores correspondientes al conjunto nacional, que sirven de base para la elaboración de los indicadores provinciales, son:

Cifras absolutas

Valores relativos

Gasto anual (millones de pesetas):

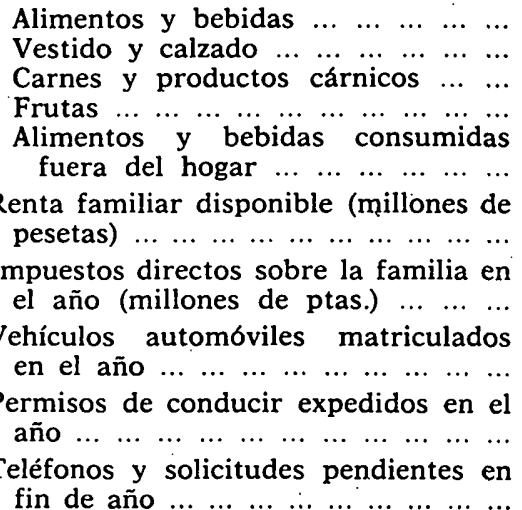

\begin{tabular}{|c|c|}
\hline 1.098 .199 & Miles de ptas/persona ... \\
\hline 191.193 & $\operatorname{Idem} \quad \begin{array}{llllllll} & \ldots & \ldots & \ldots & \ldots & \ldots & \ldots & \ldots\end{array}$ \\
\hline 274.345 & $\begin{array}{lllllllll} & & \ldots & \ldots & \ldots & \ldots & \ldots & \ldots & \ldots\end{array}$ \\
\hline 65.620 & $\begin{array}{llllllll} & \text { Idem } \\
& \ldots & \ldots & \ldots & \ldots & \ldots & \ldots & \ldots\end{array}$ \\
\hline 152.879 & $\begin{array}{lllllllll}\text { Idem } & \ldots & \ldots & \ldots & \ldots & \ldots & \ldots & \ldots\end{array}$ \\
\hline 3.121 .252 & $\operatorname{Idem} \quad \begin{array}{lllllll}\ldots & \ldots & \ldots & \ldots & \ldots & \ldots & \ldots\end{array}$ \\
\hline 68.590 & Miles ptas $/ 10$ habitantes. \\
\hline 700.943 & Por 1.000 habitantes $\ldots \ldots$ \\
\hline 954.912 & $\begin{array}{llllllll}\text { Idem } & \ldots & \ldots & \ldots & \ldots & \ldots & \ldots & \ldots\end{array}$ \\
\hline 6.541 .816 & $\begin{array}{llllll}\ldots & \ldots & \ldots & \ldots & \ldots & \ldots \\
\end{array}$ \\
\hline
\end{tabular}

El indicador de grupo del Anexo expresa los valores más altos, que corresponden a las 15 Provincias siguientes:- a) Santander, Vascongadas y Navarra, Logroño, Zaragoza y Lérida. b) Orense, Bar- 
celona, Baleares y Tarragona. c) Valencia y Alicante; y d) Madrid. Distribución básicamente similar a la de los anteriores indicadores.

En su visión global es desalentador que 35 Provincias se hallen situadas por debajo del consumo medio nacional, y ello indica la injusta distribución provincial de la renta y de la riqueza en el país.

Los gastos de consumo dependen "de la capacidad de gasto de los hogares, de su propensión al consumo y del nivel de precios", características las dos últimas difícil de aislar. Estos gastos son, a su vez, un indicador valioso del nivel de vida de la población. Las Provincias con mayor gasto total anual de consumo por persona son, con pequeñas variantes, las enumeradas en el indicador de grupo. En vestido y calzado destacan, además, La Coruña y Oviedo, Alicante y Valladolid y Segovia. El consumo de carne y sus productos, en tasas superiores a la media nacional, está más generalizado, ampliándose a otras Provincias típicamente ganaderas en ambas Mesetas y el Valle del Ebro. Se da la paradoja de que Lugo, gran productora de carne, ofrece un consumo muy bajo. El consumo de frutas resulta preponderante en Alava y Vizcaya; Gerona, Barcelona, Baleares, Tarragona y Murcia; Málaga, Sevilla y Cádiz; Canarias y Madrid. Y el gasto de consumo fuera del hogar se pone de manifiesto principalmente en Santander, Vascongadas y Navarra, Barcelona y Valencia, Málaga, Santa Cruz de Tenerife, Madrid y Valladolid. Común denominador de estos consumos es la ubicación de casi todas las Provincias, con tasas elevadas en la mitad septentrional de la Península.

La renta familiar disponible es paralela al gasto total de consumo y se manifiesta, por su importancia, en las mismas Provincias que figuran en el indicativo de grupo; y lo propio se puede decir, en líneas generales, de la distribución de los impuestos directos sobre la familia en sus valores más elevados, de los vehiculos matriculados y de los teléfonos instalados. La expedición de permisos de conducir presenta un carácter de mayor generalidad y afecta, en sus más altas tasas, a 20 Provincias, entre las que figuran como básicas las aludidas en el indicador de grupo.

\section{Promoción cultural}

Las variables que se tienen en cuenta en este grupo y los valores correspondientes al conjunto nacional, que sirven de base para la elaboración de los indicadores provinciales, son: 


\section{Cifras absolutas}

Personas que saben leer y escribir ...

Que están cursando estudios

25.100 .901

7.370 .956

Que están cursando estudios universitarios

Que no están cursando estudios y tienen título universitario o de técnico superior
202.724

311.453

Por 1.000 habitantes mayores de diez años...

912,3

218,1

$\mathbf{6 0 , 0}$

92,1

Centros de educación preescolar:

Puestos escolares

888.137

Por 1.000 habitantes

Por 10.000 habitantes ...

Idem

25,6

825.605

Por 1.000 habitantes ... ...

23,8

Centros de E. G. B.:

Puestos escolares

Idem

Alumnos matriculados

5.577 .163

Idem

160,3

4.926.641

Idem

Bachillerato:

Puestos escolares

1.223 .925

Idem

35,2

Alumnos matriculados

1.008 .596

Idem

Formación profesional:

Puestos escolares

332.528

Idem

9,6

Alumnos matriculados

204.771

Idem

Alumnos matriculados en Escuelas Universitarias del Profesorado de Educación General Básica ... ... ...

Gasto anual de las familias en enseñanza (millones de ptas.) ... .........

Prestatarios inscritos en las bibliote$\begin{array}{lllllllllllll} & \text { cas } & \ldots & \ldots & \ldots & \ldots & \ldots & \ldots & \ldots & \ldots & \ldots & \ldots & \ldots\end{array}$

Documentos prestados, en original, por las bibliotecas en el año

44.080 Por 10.000 habitantes ...

$\begin{array}{lllll}55.299 & \text { Pesetas/habitantes } & \ldots & \ldots & 1.591,8\end{array}$

1.334.214 Por 1.000 habitantes ... ... $\quad 38,4$

$\begin{array}{llllllllll}6.463 .794 & \text { Idem } & \ldots & \ldots & \ldots & \ldots & \ldots & \ldots & \ldots & 186,1\end{array}$

El indicador de grupo, que figura en el Anexo, difiere parcialmente de los anteriores. Los valores más altos en este caso corresponden a: a) Oviedo, Santander, Vascongadas, Navarra, Logroño, Zaragoza, Huesca y Lérida. b) León, Salamanca, Palencia, Burgos, Valladolid, Segovia y Soria; y $c$ ) Madrid y Guadalajara. La novedad viene dada por las Provincias del Valle del Duero.

La promoción cultural «es indispensable para la participación de la persona en la vida económica y social y condicionante de sus 
ingresos y de su nivel de vida, así como de su capacidad de disfrute de los bienes más elevados de la cultura".

Es evidente que hay una mayor promoción cultural en la zona Norte que en la zona Sur del país. Es de subrayar que la Provincia de Soria figure a la cabeza, por encima de algunas Provincias industrializadas, juntamente con las Vascongadas y Madrid.

Las personas que saben leer y escribir sobresalen en 24 Provincias, con tasas superiores al promedio nacional. Son: La Coruña y de Oviedo a Guipúzcoa en la orla cantábrica; todas las del Valle del Duero; las del Valle del Ebro, excepto Teruel; más Barcelona y Gerona. La mitad Norte de la Península se ha caracterizado siempre por la alfabetización masiva de sus habitantes.

Las personas que están cursando estudios son más numerosas en las Provincias de más alta natalidad, por cuanto disponen de un mayor volumen de población en edad escolar. Las 24 Provincias con tasas superiores a la media nacional comprenden: a) Vascongadas y Navarra. b) Burgos, León, Palencia, Salamanca, Segovia, Soria y Valladolid. c) Madrid, Albacete y Extremadura. d) Murcia y Andalucía, menos Málaga y Huelva; y e) Canarias. Se observa cómo se extiende la promoción cultural al Centro y Sur del país.

Las personas que cursan o han cursado estudios universitarios sobresalen en una zona geográficamente más restringida, localizada con preferencia al Norte del país. Comprende las siguientes Provincias: Madrid, Vascongadas, Navarra y Zaragoza; Salamanca, Valladolid y Segovia; Barcelona y Valencia, y Granada. En su mayor. parte son Provincias universitarias.

La enseñanza en centros de educación preescolar presenta una falta de homogeneidad entre las distintas Provincias; para corregirla sería necesario conocer la población en edad preescolar de cada una de ellas. La educación general básica es más homogénea en su distribución, generalizada en todo el país; hasta 36 Provincias ofrecen tasas superiores a la media nacional, y los valores de las restantes Provincias se sostienen, con alguna excepción, por encima del 90 por 100 . Lo propio puede decirse de los estudios de bachillerato, que se desarrollan principalmente en 25 Provincias, situadas al Norte del país.

La dispersión geográfica de los centros docentes de todos los niveles determina que los puestos escolares no se cubran totalmente con los alumnos matriculados, especialmente en los Municipios 
no capitales. La diferencia es más acusada con referencia a la formación profesional, con un evidente déficit de alumnado.

El volumen de gastos de las familias en enseñanza está en razón directa con su capacidad de gasto y depende, además, del grado de gratuidad y del predominio de la enseñanza pública o privada. Es evidente que ha habido una eclosión de la enseñanza, a todos los niveles, en la mayoría de los hogares españoles, en los que se considera la enseñanza como una necesidad de primer orden, y también es cierto que no hay suficiente número de puestos gratuitos para satisfacer adecuadamente esta demanda social. El gasto anual medio de 1.592 pesetas por persona puede considerarse elevado, dado que el alumnado de enseñanza superior es aún escaso. Los mayores gastos se registran en un número reducido de Provincias: Santander, Vascongadas, Navarra y Zaragoza; Barcelona y Tarragona; Madrid, Segovia y Soria. La actividad bibliotecaria debe considerarse como complementaria de la docente.

\section{CONVIVENCIA SOCIAL}

Las variables que se tienen en cuenta en este grupo como representativas de los complejos problemas de la convivencia social y los valores correspondientes al conjunto nacional, que sirven de base para la elaboración de los indicadores provinciales, son:

Cifras absolutas

Tribunales Tutelares de Menores. Expedientes abiertos y reabiertos ......

Delitos apreciados en Audiencias y Juzgados

Sentencias condenatorias en Juzgados municipales, comarcales y de paz ...

Asuntos de jurisdicción contenciosa resueltos

Asuntos de jurisdicción contenciosa despachados por sentencia según $\begin{array}{lllllll}\text { juicio ejecutivo } & \ldots & \ldots & \ldots & \ldots & \ldots & \ldots\end{array}$

Juicios incoados, verbales, de cognición y de arrendamientos rústicos y urbanos en los Juzgados municipa. les, comarcales y de paz ... ... ... ...

Suicidios consumados y tentativas (promedio del quinquenio) $\ldots \ldots \ldots$

\section{Valores relativos}

17.813 Por 100.000 habitantes ...

$\begin{array}{llllllllll}49.850 & \text { Idem } & \ldots & \ldots & \ldots & \ldots & \ldots & \ldots & \ldots & 143,5\end{array}$

$\begin{array}{llllllllll}126.801 & \text { Idem } & \ldots & \ldots & \ldots & \ldots & \ldots & \ldots & \ldots & 365,0\end{array}$

$\begin{array}{lllllllllll}86.907 & \text { Idem } & \ldots & \ldots & \ldots & \ldots & \ldots & \ldots & \ldots & 250,2\end{array}$

$\begin{array}{llllllllll}43.934 & \text { Idem } & \ldots & \ldots & \ldots & \ldots & \ldots & \ldots & \ldots & 126,5\end{array}$

$\begin{array}{lllllllllr}107.317 & \text { Idem } & \ldots & \ldots & \ldots & \ldots & \ldots & \ldots & \ldots & 308,9\end{array}$

$\begin{array}{lllllllllll}1.726 & \text { Idem } & \ldots & \ldots & \ldots & \ldots & \ldots & \ldots & \ldots & 5,3\end{array}$ 
Cifras absolutas

Valores relativos

Protestos de documentos de giro ....... Accidentes de circulación con vícti$\begin{array}{llllllllllll}\operatorname{mas} & \ldots & \ldots & \ldots & \ldots & \ldots & \ldots & \ldots & \ldots & \ldots & \ldots & \ldots\end{array}$ Acogidos en establecimientos benéfi$\cos$
2.782.018 Por 1.000 habitantes .......

62.614 Por 10.000 habitantes ...

112.648 Idem
80,1

32,4

En el indicado grupo del Anexo figuran los valores más altos en relación con el promedio nacional, que en este caso deben interpretarse en sentido negativo, por cuanto suponen una agravación de los problemas de convivencia, propios de las grandes urbes, de los núcleos industriales o en vías de industrialización y de las zonas deprimidas agrarias, con deficiente equipamiento social, hacinamiento, insuficiencia económica y bajo nivel cultural. En el sentido expuesto destacan las Provincias: a) Santander, Alava, Logroño y Zaragoza. b) Gerona, Barcelona, Baleares y Alicante. c) Andalucía, salvo Cádiz y Jaén. d) Burgos, Valladolid y Palencia. e) Las Palmas; y $f$ ) Madrid.

La delincuencia juvenil y la protección del menor, medidas por las actividades de los Tribunales Tutelares de Menores en el ejercicio de sus facultades reformadora y protectora, presenta sus tasas más elevadas en 25 Provincias, entre ellas las Vascongadas y Logroño, la mayor parte del Valle del Duero, varias de la Submeseta Sur, Andalucía y Canarias.

La delincuencia general, expresada por el número de delitos apreciados en las Audiencias provinciales y Juzgados de Primera Instancia, cubre, en sus tasas más altas, 20 Provincias: Pontevedra, Oviedo y Santander; Alava, Logroño y Zaragoza; Barcelona y Baleares; la Región valenciana; Almería y Huelva; Canarias; Valladolid, Palencia y Soria, y Madrid y Cuenca, con representación en todas las Regiones.

Las sentencias condenatorias dictadas en juicios de faltas, expresión de la delincuencia menor, ofrecen una más amplia distribución, que alcanza a 23 Provincias: Oviedo, Santander y Vizcaya; Alava, Logroño, Lérida y Teruel; Gerona; Andalucía, excepto Almería; Burgos, León y Palencia; Madrid, Toledo, Ciudad Real, Cáceres y Albacete. Esta delincuencia tiene menos peso en Barcelona, Baleares y la Región valenciana; pero mayor entidad en la Submeseta Sur y Andalucía. La litigiosidad, en sus valores más altos, que- 
da restringida a 11 Provincias: Barcelona, Baleares, Valencia, Alicante, Sevilla y Málaga; Canarias; Zaragoza, Valladolid y Madrid, en las que las concentraciones de población y el tráfico mercantil derivado de su actividad económica dan lugar a cuestiones que han de dirimirse ante los Tribunales. Los protestos de documentos de giro presentan también, en sus valores máximos, una distribución similar.

El fenómeno del suicidio tiende a decrecer en sus cifras absolutas, aunque, como síntoma psico-social, se mantiene extendido a 31 Provincias ubicadas en todas las Regiones consideradas.

La distribución provincial de los accidentes de circulación, dadas las características del tráfico, es muy amplia y poco significativa. La labor de asistencia social en los establecimientos benéficos resulta insuficiente, está determinada principalmente por la acción del Estado, de las Corporaciones locales y de la iniciativa privada y se manifiesta prácticamente en todas las Provincias, con diferencias cuantitativas de escaso valor.

\section{ESPARCIMIENTO}

Las variables que se tienen en cuenta en este grupo y los valores correspondientes al conjunto nacional, que sirven de base para la elaboración de los indicadores provinciales, son:

Valores relativos

Gasto anual de los hogares en esparcimiento, deportes y cultura (millo-

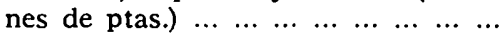

Licencias de caza y pesca en el año. Instalaciones deportivas existentes ...

Espectadores de cine en el año (miles) $\ldots \ldots \ldots \ldots \ldots \ldots \ldots$

Recaudación en salas de proyección cinematográfica en el año (miles

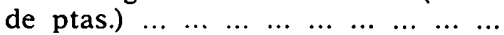

Plazas en establecimientos hoteleros.

Plazas en restaurantes $\ldots \ldots \ldots \ldots \ldots$
Cafeterías, cafés, bares y salas de

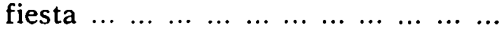

Socios de teleclubs en el trienio ... ... Espectáculos taurinos en el trienio ...

Personas que practican habitualmente deporte

\begin{tabular}{|c|c|}
\hline $\begin{array}{r}121.448 \\
1.502 .702 \\
19.558\end{array}$ & $\begin{array}{l}\text { Miles de ptas/persona } \\
\text { Por } 1.000 \text { habitantes ... } \\
\text { Por } 100.000 \text { habitantes }\end{array}$ \\
\hline 250.314 & Por habitante $\ldots . . . .$. \\
\hline $\begin{array}{r}9.819 .489 \\
731.801 \\
1.154 .728\end{array}$ & $\begin{array}{l}\text { Pesetas/habitante } \ldots \\
\text { Por } 1.000 \text { habitantes } \ldots \\
\text { Idem } \ldots \ldots \ldots \\
\end{array}$ \\
\hline $\begin{array}{r}87.321 \\
813.771 \\
12.031\end{array}$ & $\begin{array}{l}\text { Por } 10.000 \text { habitantes } \\
\text { Por } 1.000 \text { habitantes ... } \\
\text { Por } 100.000 \text { habitantes }\end{array}$ \\
\hline 4.598 .902 & $\begin{array}{l}\text { Por } 1.000 \text { habitantes } \\
\text { diez a setenta años }\end{array}$ \\
\hline
\end{tabular}
3,5 43,3 56,3 
En el indicador de grupo del Anexo figuran los valores más altos, que corresponden a las siguientes Provincias: a) Santander, Vascongadas y Navarra, Logroño, Zaragoza, Huesca y Lérida. b) Gerona, Baleares, Tarragona y Alicante. $c$ ) Las Palmas. $d$ ) El Valle del Duero; y $e$ ) Madrid, Guadalajara y Cuenca.

El «empleo del ocio» es una de las dimensiones del bienestar que recibe creciente atención en el ámbito de la política social de los países desarrollados. Salvo el gasto de los hogares, que constituye un indicador útil del «bienestar producido por el empleo del tiempo libre», los indicadores que se han tomado "no tienen clara significación en relación con el bienestar de la población ni con el grado de desarrollo de la Provincia».

El gasto de los hogares en esparcimiento, deportes y cultura, que no incluye los de vacaciones ni los ocasionados por la vivienda secundaria, presenta sus valores máximos en 15 Provincias: Santander, Vascongadas, Logroño y Zaragoza; Gerona, Barcelona, Baleares, Tarragona, Valencia y Alicante; Canarias, y Madrid, entre las cuales se hallan las calificadas como de mayor importancia demográfica y económica.

\section{Equipamiento de Los hogares}

Las variables que se tienen en cuenta en este grupo y los valores correspondientes al conjunto nacional, que sirven de base para la elaboración de los indicadores provinciales, son:

Familias que habitan en casas com-

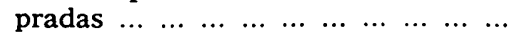
Que habitan viviendas construidas con posterioridad a 1955

4.396.271 Por 100 familias ... ... ...

Hogares que disponen de igual o mayor número de habitaciones que de $\begin{array}{lllllllllll}\text { personas } & \ldots & \ldots & \ldots & \ldots & \ldots & \ldots & \ldots & \ldots & \ldots & \end{array}$ Cuya vivienda es de $65 \mathrm{~m}^{2}$ o más ... Cuya vivienda dispone de agua corriente caliente y fría $\ldots . . . . . . . .$. Cuya vivienda dispone de baño .......

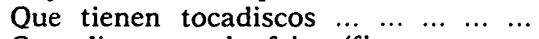
Que disponen de frigorífico $\ldots \ldots \ldots$ Que usan electricidad o gas en la co$\begin{array}{llllllllllll}\text { cina } & \ldots & \ldots & \ldots & \ldots & \ldots & \ldots & \ldots & \ldots & \ldots & \ldots & \ldots\end{array}$

Que consumen más de $50 \mathrm{kw}$. mensuales de energía eléctrica $\ldots . . . . . .$.

$\begin{array}{llllllll}3.909 .810 & \text { Idem } & \ldots & \ldots & \ldots & \ldots & \ldots & \ldots\end{array}$

5.840.289 Por 100 hogares ... ... ... 62,5

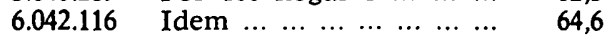

$\begin{array}{llllllllll}4.644 .212 & \text { Idem } & \ldots & \ldots & \ldots & \ldots & \ldots & \ldots & \ldots & 49,7\end{array}$

$\begin{array}{llllllllll}5.352 .546 & \text { Idem } & \ldots & \ldots & \ldots & \ldots & \ldots & \ldots & \ldots & 57,2\end{array}$

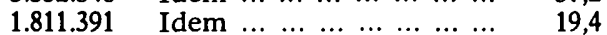

$\begin{array}{lllllllll}8.893 .401 & \text { Idem } & \ldots & \ldots & \ldots & \ldots & \ldots & \ldots & 73,7\end{array}$

$\begin{array}{llllllllll}7.963 .673 & \text { Idem } & \ldots & \ldots & \ldots & \ldots & \ldots & \ldots & \ldots & 85,2\end{array}$

$\begin{array}{llllllllll}6.333 .190 & \text { Idem } & \ldots & \ldots & \ldots & \ldots & \ldots & \ldots & \ldots & 67,7\end{array}$ 
En el indicado grupo del Anexo figuran los valores más altos, que corresponden a: Santander, Vascongadas y Navarra, Zaragoza y Lérida; Gerona, Barcelona, Baleares, Castellón, Valencia, Alicante y Murcia; Madrid y Valladolid. Distribución que difiere parcialmente de la referente al gasto de los hogares en esparcimiento.

La adquisición de la vivienda, "cuyas características y servicios, formas de acceso y ubicación no responden a las necesidades objetivas de la población", en lugar de ser un índice de satisfacción, se convierte, en cierto sentido, en un índice negativo por las trabas, ajenas a la voluntad del comprador, que la adquisición lleva consigo.

La distribución de los valores máximos, en cuanto a las características estudiadas de compra u ocupación, instalaciones, disponibilidad de tocadiscos o frigorífico y consumo de energía eléctrica, coinciden, en general, con las Provincias enumeradas en el indicador de grupo, si bien, respecto a la superficie de la vivienda, número de habitaciones y al uso de electricidad o gas en la cocina, se extiende a mayor número de Provincias.

\section{NIVEL CONJUNTO DE BIENESTAR SOCIAL}

Se han transcrito las tablas elaboradas referidas a cada indicador de grupo y a las variables contempladas en cada uno de ellos con respecto al conjunto nacional, y se ha expuesto, en forma a veces reiterativa y analizando la distribución de unos y otras, en la forma más concisa posible, considerando, en primer término, las Provincias litorales y marítimas y, en segundo lugar, las interiores.

Nos referimos ahora al indicador conjunto, contenido en el Anexo, que permite establecer "una valoración comparativa entre los niveles alcanzados en las distintas Provincias y en el conjunto nacional».

Como se indica en la monografía, del examen de los datos comentados parece deducirse, en primer lugar, que el bienestar «es mayor en la zona Norte que en la Sur» y que "en las Provincias del litoral hay un mayor bienestar que en las del interior».

Las Provincias que aparecen con un indicador superior a 100 son 15. Salvo Madrid y Valladolid, las restantes se hallan situadas en el polígono limitado por los vértices de Santander, Alicante, Ba- 
leares y Gerona, aunque dentro de dicho polígono hay Provincias con valores inferiores al nacional.

Tal vez no hay en España «zonas de bienestar», sino, más bien, "focos de bienestar", es decir, "puntos en los que se dan una serie de condiciones, de servicios y de infraestructura que hace la existencia más agradable y cómoda y, por ello, atraen a grandes masas de población».

Los focos de bienestar básicos son: Madrid, las Vascongadas, Barcelona y Valencia. Alrededor de las Vascongadas se hallan, como focos por ellas influidos, Santander, Navarra y Logroño. Barcelona irradia su influencia sobre Gerona, Tarragona y Baleares e influye también, de cierta manera, sobre Valencia y Alicante. Madrid no irradia sobre las Provincias limítrofes, pero de sus conexiones con el Norte y con Barcelona surge su influencia sobre Valladolid y Zaragoza, respectivamente. Las 35 Provincias restantes pueden clasificarse como pertenecientes, con distinta gradación, a una amplia zona de peor bienestar relativo, dentro de la que pueden distinguirse dos subzonas peor dotadas: la gallega y la andaluzaextremeña-manchega, con prolongaciones hacia los Valles del Duero y Ebro.

Los focos de bienestar están altamente industrializados, considerando incluida la industria turística. Tal es el caso de Vizcaya, Guipúzcoa, Barcelona y Madrid. Otros se hallan en un franco proceso de industrialización, e incluso en aquellos que disponen de una riqueza agrícola tradicional, su condición de foco viene deter. minada más por su actual industrialización. 
IV. ANEXO

\begin{tabular}{|c|c|c|c|c|c|c|c|c|c|}
\hline \multirow[b]{2}{*}{ Provincias } & \multirow[b]{2}{*}{$\begin{array}{l}\text { INDICA- } \\
\text { DOR DE } \\
\text { CONJUNTO }\end{array}$} & \multicolumn{8}{|c|}{ INDICADORES DE GRUPO } \\
\hline & & $\begin{array}{c}1 \\
\text { Pobla- } \\
\text { ción }\end{array}$ & $\begin{array}{c}2 \\
\text { Atencio- } \\
\text { nes sa- } \\
\text { nitarias }\end{array}$ & $\begin{array}{c}3 \\
\text { Activi- } \\
\text { dad la- } \\
\text { boral }\end{array}$ & $\begin{array}{c}4 \\
\text { Capaci- } \\
\text { dad de } \\
\text { consumo }\end{array}$ & $\begin{array}{c}5 \\
\text { Promo- } \\
\text { ción } \\
\text { cultural }\end{array}$ & $\begin{array}{c}6 \\
\text { Convi- } \\
\text { vencia } \\
\text { social }\end{array}$ & $\begin{array}{c}7 \\
\text { Espar- } \\
\text { cimiento }\end{array}$ & $\begin{array}{c}8 \\
\text { Equipa- } \\
\text { miento } \\
\text { hogares }\end{array}$ \\
\hline 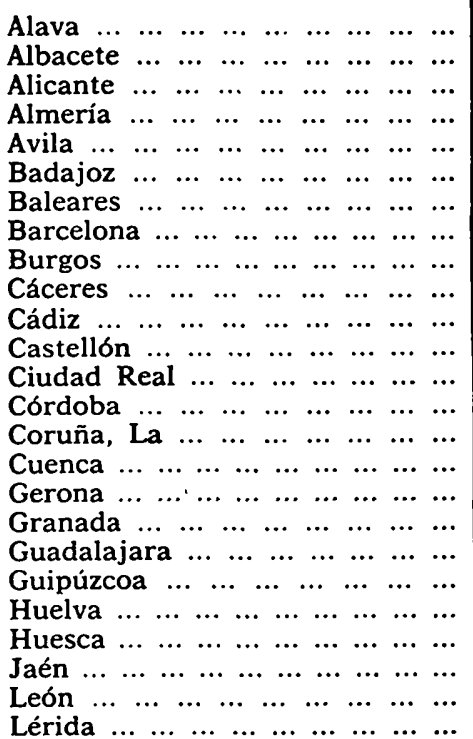 & $\begin{array}{r}104,3 \\
95,8 \\
101,7 \\
95,5 \\
94,8 \\
94,1 \\
100,6 \\
103,3 \\
98,1 \\
94,5 \\
97,6 \\
99,8 \\
95,3 \\
94,3 \\
97,9 \\
94,4 \\
102,0 \\
94,6 \\
97,2 \\
104,4 \\
94,4 \\
97,9 \\
94,3 \\
97,6 \\
99,6\end{array}$ & $\begin{array}{r}105,1 \\
91,9 \\
102,9 \\
99,2 \\
88,7 \\
88,5 \\
98,2 \\
111,4 \\
94,1 \\
89,3 \\
105,3 \\
95,3 \\
89,6 \\
93,7 \\
97,3 \\
83,4 \\
98,3 \\
94,9 \\
80,6 \\
104,3 \\
95,7 \\
86,2 \\
91,1 \\
91,9 \\
92,9\end{array}$ & $\begin{array}{r}100,5 \\
93,6 \\
99,8 \\
92,2 \\
94,3 \\
94,1 \\
99,6 \\
105,1 \\
97,4 \\
95,3 \\
97,8 \\
100,5 \\
94,9 \\
92,6 \\
98,5 \\
91,1 \\
99,7 \\
95,4 \\
100,1 \\
103,3 \\
94,6 \\
99,2 \\
93,2 \\
95,5 \\
95,2\end{array}$ & $\begin{array}{r}102,8 \\
98,1 \\
104,9 \\
98,1 \\
94,2 \\
96,8 \\
101,5 \\
99,8 \\
98,8 \\
97,5 \\
95,8 \\
103,8 \\
103,2 \\
96,7 \\
104,3 \\
95,9 \\
101,9 \\
92,2 \\
89,0 \\
96,1 \\
94,6 \\
98,5 \\
95,1 \\
96,3 \\
101,5\end{array}$ & $\begin{array}{r}104,7 \\
90,9 \\
102,5 \\
90,6 \\
87,6 \\
88,6 \\
107,3 \\
111,9 \\
93,9 \\
84,1 \\
94,2 \\
98,9 \\
90,1 \\
91,3 \\
94,5 \\
88,8 \\
105,0 \\
87,7 \\
94,2 \\
107,2 \\
92,0 \\
95,7 \\
86,0 \\
93,5 \\
100,4\end{array}$ & $\begin{array}{r}108,9 \\
98,0 \\
96,4 \\
97,1 \\
94,8 \\
94,9 \\
95,8 \\
96,5 \\
104,6 \\
97,4 \\
95,3 \\
95,3 \\
95,2 \\
91,0 \\
94,1 \\
96,8 \\
96,4 \\
98,8 \\
103,8 \\
108,4 \\
92,5 \\
100,5 \\
96,0 \\
105,2 \\
100,6\end{array}$ & $\begin{array}{r}104,2 \\
98,3 \\
102,3 \\
105,7 \\
93,4 \\
94,6 \\
113,5 \\
103,1 \\
101,8 \\
92,9 \\
97,9 \\
97,3 \\
97,3 \\
101,3 \\
91,5 \\
91,6 \\
101,2 \\
104,9 \\
96,1 \\
93,5 \\
103,0 \\
96,4 \\
94,3 \\
98,3 \\
97,1\end{array}$ & $\begin{array}{r}109,2 \\
98,4 \\
103,3 \\
95,2 \\
104,4 \\
95,9 \\
113,6 \\
99,1 \\
105,4 \\
98,3 \\
96,0 \\
99,5 \\
93,7 \\
95,4 \\
95,7 \\
100,3 \\
110,0 \\
94,7 \\
111,6 \\
103,3 \\
93,9 \\
103,3 \\
93,1 \\
102,6 \\
102,8\end{array}$ & $\begin{array}{r}107,2 \\
93,5 \\
105,7 \\
97,2 \\
87,4 \\
88,9 \\
102,2 \\
105,8 \\
92,1 \\
86,6 \\
94,2 \\
102,7 \\
93,2 \\
95,3 \\
90,3 \\
90,4 \\
105,6 \\
96,8 \\
94,5 \\
106,0 \\
94,7 \\
96,4 \\
94,1 \\
94,2 \\
100,1\end{array}$ \\
\hline
\end{tabular}




\begin{tabular}{|c|c|c|c|c|c|c|c|c|c|}
\hline \multirow[b]{2}{*}{ Provincias } & \multirow[b]{2}{*}{$\begin{array}{c}\text { INDICA- } \\
\text { DOR DE } \\
\text { CONJUNTO }\end{array}$} & \multicolumn{8}{|c|}{ INDICADORES DE GRUPO } \\
\hline & & $\begin{array}{c}1 \\
\text { Pobla- } \\
\text { ción }\end{array}$ & $\begin{array}{c}2 \\
\text { Atencio- } \\
\text { nes sa- } \\
\text { nitarias }\end{array}$ & $\begin{array}{c}3 \\
\text { Activi- } \\
\text { dad la- } \\
\text { boral }\end{array}$ & $\begin{array}{c}4 \\
\text { Capaci- } \\
\text { dad de } \\
\text { consumo }\end{array}$ & $\begin{array}{c}5 \\
\text { Promo- } \\
\text { cion } \\
\text { cultural }\end{array}$ & $\begin{array}{c}6 \\
\text { Convi- } \\
\text { vencia } \\
\text { social }\end{array}$ & $\begin{array}{c}7 \\
\text { Espar- } \\
\text { cimiento }\end{array}$ & $\begin{array}{c}8 \\
\text { Equipa- } \\
\text { miento } \\
\text { hogares }\end{array}$ \\
\hline 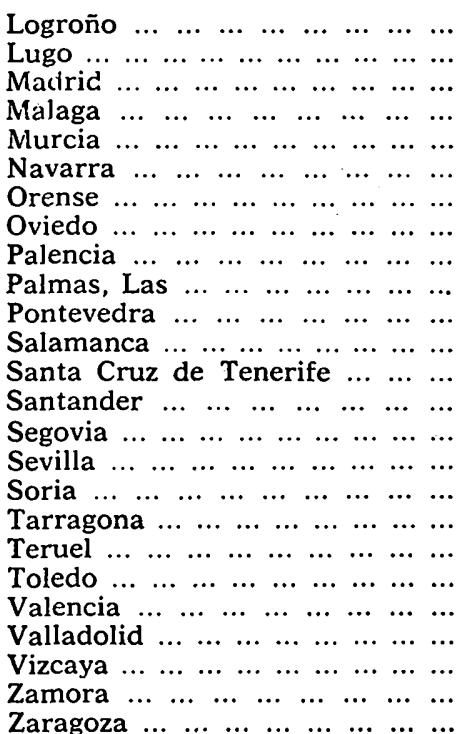 & $\begin{array}{r}100,9 \\
92,2 \\
106,5 \\
96,4 \\
99,9 \\
102,6 \\
94,9 \\
98,7 \\
97,6 \\
99,4 \\
97,6 \\
97,4 \\
98,0 \\
101,3 \\
98,4 \\
96,9 \\
98,1 \\
101,4 \\
94,1 \\
96,6 \\
102,1 \\
100,2 \\
105,8 \\
94,2 \\
101,3\end{array}$ & $\begin{array}{r}97,2 \\
85,8 \\
113,6 \\
99,7 \\
100,1 \\
98,6 \\
87,0 \\
97,1 \\
90,1 \\
107,2 \\
99,9 \\
92,3 \\
104,3 \\
100,4 \\
87,8 \\
103,2 \\
82,3 \\
99,0 \\
80,3 \\
91,7 \\
103,1 \\
102,6 \\
108,6 \\
87,8 \\
99,6\end{array}$ & $\begin{array}{r}103,5 \\
88,5 \\
104,4 \\
98,5 \\
98,3 \\
103,8 \\
89,3 \\
103,2 \\
99,0 \\
95,7 \\
95,2 \\
98,1 \\
96,5 \\
104,0 \\
97,1 \\
10,2 \\
99,0 \\
103,1 \\
98,1 \\
96,7 \\
104,0 \\
98,6 \\
104,0 \\
91,6 \\
105,7\end{array}$ & $\begin{array}{r}99,3 \\
101,2 \\
107,0 \\
96,5 \\
100,4 \\
95,4 \\
108,6 \\
96,6 \\
95,8 \\
99,7 \\
104,1 \\
97,2 \\
101,0 \\
99,1 \\
96,3 \\
95,7 \\
92,8 \\
101,4 \\
91,0 \\
99,0 \\
103,0 \\
93,2 \\
103,5 \\
95,4 \\
97,3\end{array}$ & $\begin{array}{r}101,0 \\
84,2 \\
112,2 \\
94,5 \\
97,4 \\
102,5 \\
92,1 \\
97,6 \\
92,5 \\
95,9 \\
95,1 \\
88,1 \\
94,8 \\
101,2 \\
94,5 \\
95,2 \\
93,9 \\
101,4 \\
90,3 \\
92,8 \\
101,6 \\
99,9 \\
108,0 \\
89,4 \\
100,4\end{array}$ & $\begin{array}{r}102,9 \\
90,4 \\
106,6 \\
93,9 \\
99,3 \\
104,7 \\
89,3 \\
100,8 \\
103,8 \\
97,3 \\
94,1 \\
105,0 \\
92,4 \\
102,8 \\
105,5 \\
96,8 \\
109,8 \\
99,9 \\
94,5 \\
95,0 \\
99,7 \\
108,0 \\
107,0 \\
96,9 \\
101,7\end{array}$ & $\begin{array}{r}103,1 \\
90,8 \\
103,4 \\
108,2 \\
95,8 \\
96,3 \\
87,4 \\
96,9 \\
102,4 \\
102,5 \\
93,4 \\
94,0 \\
99,8 \\
102,3 \\
95,5 \\
105,4 \\
99,6 \\
97,3 \\
91,9 \\
93,4 \\
99,0 \\
99,1 \\
95,6 \\
102,4 \\
102,6\end{array}$ & $\begin{array}{r}106,9 \\
95,9 \\
103,5 \\
99,5 \\
96,9 \\
105,9 \\
94,2 \\
96,1 \\
105,6 \\
101,8 \\
94,8 \\
100,9 \\
97,1 \\
104,7 \\
107,1 \\
93,4 \\
113,8 \\
103,9 \\
99,4 \\
97,4 \\
98,9 \\
104,1 \\
101,4 \\
101,1 \\
105,2\end{array}$ & $\begin{array}{r}99,7 \\
82,1 \\
107,6 \\
96,9 \\
102,5 \\
106,2 \\
86,0 \\
95,1 \\
96,2 \\
99,8 \\
91,0 \\
91,6 \\
97,6 \\
100,3 \\
94,6 \\
96,4 \\
92,7 \\
99,9 \\
90,8 \\
93,6 \\
105,6 \\
110,0 \\
86,8 \\
100,4 \\
103,2\end{array}$ \\
\hline
\end{tabular}




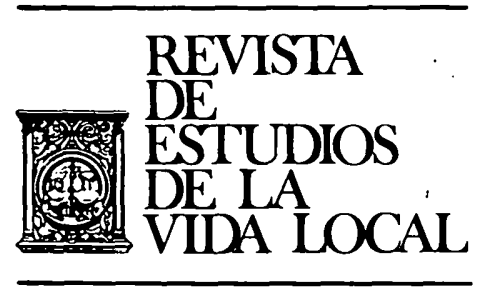

\section{JURISPRUDENCIA}


REVL-1977, núm: 194. BALLESTER ROS, IGNACIO. NOTAS SOBRE LOS NIVELES PROVINCIALE... REVL-1977, núm. 194. BALLESTER ROS, IGNACIO. NOTAS SOBRE LOS NIVELES PROVINCIALE... 